\title{
TRABAJO PRECARIO Y DESEMPLEO OCULTO: LA ESTRUCTURA OCUPACIONAL EN SANTIAGO DEL ESTERO, ARGENTINA*
}

\author{
Carlos Virgilio Zurita**
}

La imagen emblemática del trabajador informal es la del vendedor ambulante.

Sin embargo, el mundo del trabajo precario no se agota en esa metáfora, sino que la trasciende vastamente: su realidad es multiforme y, a veces, esquiva. Asume diferentes rostros; de mujeres, niños, ancianos. de jefes de familia y población secundaria, de iletrados y no tanto: más allá del género. de la edad, de la ocupación. Un caleidoscopio social de la pobreza.

Porque el desarrollo capitalista, que estructuralmente ha tendido a la modernización - esto es, la formalización — de las relaciones sociales de producción no ha operado, por diversas razones, de una manera similar en contextos económicos diferentes. Si bien en los países avanzados significó la homogenización del sistema productivo y de la estructura laboral, en las regiones periféricas ha implicado, simultáneamente, instalación de la modernidad y afianzamiento del atraso.

En Europa y los EE.UU., el trabajo informal, históricamente, decrece - los vendedores de hot dogs en Broadway o de castañas asadas en los alrededores del Louvre son, apenas, nostálgicas supervivencias-; en América Latina, en cambio, y sobre todo a partir de la década del 60, merced a la crisis del modelo sustitutivo de importaciones y a una particular dinámica poblacional en la que convergieron altos diferenciales de fecundidad y fuertes migraciones rural-urbanas, importantes contingentes se trasladaron hacia las ciudades: al no poder ser absorbidos plenamente por el sector formal de la economía, en gran parte engrosaron el contingente urbano de reserva ocupacional. Para la pauta de desarrollo vigente, eran ( $y$ son) mano de obra redundante.

Clausurados, entonces, los accesos a puestos de trabajo formales encontraron - 0 inventaron, lo que para el caso es lo mismo- caminos para el ingreso a un mundo laboral sui géneris cuya lógica no es la eficiencia sino la subsistencia y cuyo procedimiento de constitución prototípico es el autoempleo. Estas dos notas, entre otras, son características sustantivas de lo que se conoce como sector informal urbano (SIU) ${ }^{\perp}$.

* Este trabajo fue realizado durante la permanencia del autor como investigador visitante en el Centro de Estudios Sociológicos de El Colegio de México.

*: Departamento de Sociología, Universidad Nacional de Santiago del Estero/Consejo Nacional de Investigaciones Científicas y Técnicas (CONICET)

I Diversas definiciones del sector informal han sido proporcionadas por distintos autores. Inclusive la denominación misna del sector ha sido objeto de diferentes propuestas: así, se lo ha mencionado como "sector autónomo" (OIT), "sector no organizado" (Pereyra y Zink). sector "no protegido" (Merrick), "economía tipo bazar" (Geertz), como un "modo de producción de mercaderías menores" (Mc Ewen Scott). y se ha hablado de un sector "cuasi formal" instersticial entre el formal y el informal (Sánchez, Palmieri y Ferrero). Aspectos centrales de la cuestión son analizados en el ya clásico estudio de Dagmar Raczinky Sector informal: Interrogantes y controversias, CIEPLAN, 1978, por Fernando Cortés en La informalidad del sector informal extraoficial. FLACSO/México, 1988, y por Bélisie (1992). Una sugestiva discusión sobre 
La estructura y funcionamiento del sıu en las áreas metropolitanas —básicamente capitales de países - de América Latina ha sido ya suficientemente analizada. No acontece otro tanto con la realidad laboral de las ciudades intermedias —entre 100 y 500 mil habitantes, determinando márgenes convencionales-; son relativamente escasos los estudios e indagaciones al respecto. Sin embargo, por diversas razones (la óptima planificación de los recursos económicos, la adecuada formulación de políticas sociales, etc.) interesa avanzar en el análisis de la estructura social de tales ciudades de tamaño medio que, además, al menos en el caso de Argentina, poseen una reveladora significación: un peso social y demográfico considerable.

En las siguientes páginas, que constituyen una versión sintética y actualizada de un trabajo más extenso sobre el tema (Zurita, 1988), se presentan algunas acotaciones sobre la estructura ocupacional de Santiago del Estero, a partir de información proveniente de la Encuesta Permanente de Hogares (INDEC/EPH) y de entrevistas a trabajadores informales.

\section{EL EMPLEO EN UNA SOCIEDAD TRADICIONAL}

De Santiago del Estero se postula que se trata de una región deprimida en el seno de un país moderno. En realidad, el noroeste, el área donde se encuentra situada, es la zona más tradicional y, todavía, más ruralizada de la Argentina.

La capital de la provincia es la ciudad más antigua del país, y ha sido llamada "madre de ciudades" porque de ella partieron corrientes colonizadoras que fundaron varias de las ciudades más importantes de la actual Argentina.

Su historia es la crónica de un largo deterioro: de ocupar un rol particularmente importante durante el período virreinal, pasa a desempeñar una función periférica constante a partir de la incorporación — hacia 1880 — de la economía argentina al sistema de comercio mundial. Más aún, no sólo pierde peso político y su economía se estanca sino que, desde las primeras décadas del presente siglo, se ve afectada por un hondo y progresivo proceso de descapitalización humana, producido por la erosión migratoria hacia las zonas industrializadas del país. Según el Censo de población de 1991 cerca de medio millón de santiagueños residen permanentemente fuera de su provincia de origen.

En otro texto (Tasso y Zurita, 1981) se ofrece una perspectiva relativamente detallada de la estructura social de la provincia.

Ahora sólo interesa recordar la pauta básica de la configuración del empleo provincial entre 1947 y 1990. Como puede verse en el Cuadro 1, la provincia exhibe las notas paradigmáticas de las regiones subdesarrolladas: alta ruralidad y muy bajos niveles de industrialización.

Tendencialmente, la declinación en el período considerado de la ocupación en la rama I (Agricultura, silvicultura, etc.) más que significar la modernización de las activida des rurales resulta expresiva de la crisis del sector forestal que en su momento fue un gran demandante de empleo. En la misma perspectiva, la disminución laboral en la industria

: fundamentos teóricos de la informalidad en América Latina (¿se trata de una forma de utilización laboral o se trata de Lin min de producir?) entre Portes y Tokman, se desarrolla en Estudios Sociológicos, Números 13 y 16, El Colegio de Mévice. 
TRABAJO PRECARIO Y DESEMPLEO OCULTO: LA ESTRUCTURA OCUPACIONAL EN...

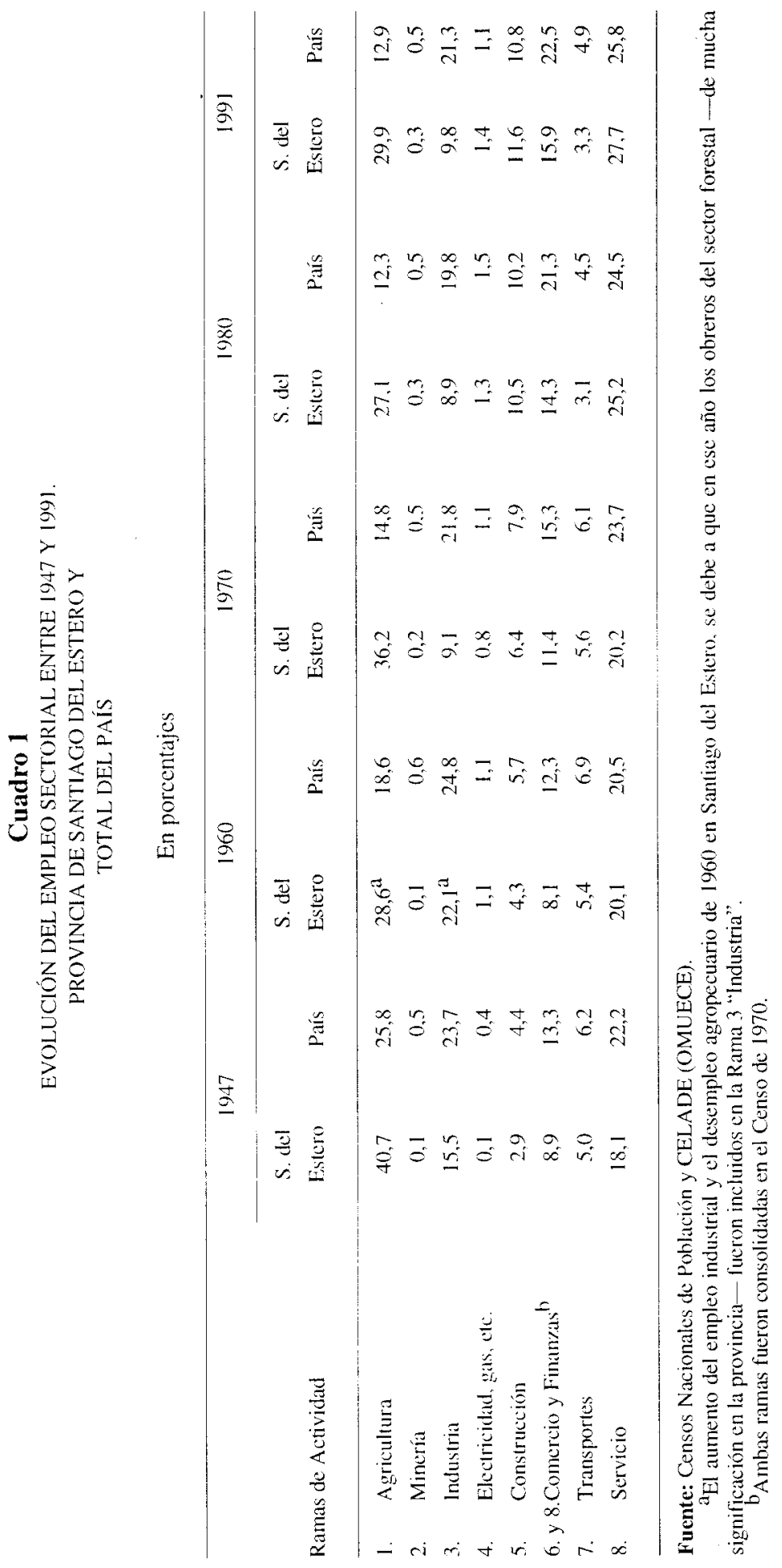




\section{REVISTA DE SOCIOLOGÍA}

supone, no la liberación de puestos de trabajo por aumento de la productividad, sino el colapso de establecimientos de naturaleza semiartesanal. Por su parte, la relevancia del empleo en servicios, especialmente en la rama 9, no implica "terciarización moderna" sino que se sustenta, sobre todo, en la hipertrofia del sector público y en la supervivencia de servicios tradicionales como el doméstico.

El área urbana en la que concentramos nuestro estudio comprende unos doscientos mil habitantes; las opciones ocupacionales de esa población pueden advertirse en el Cuadro 2. Con fines comparativos, se presenta -además de Santiago del Esteroinformación sobre otras cuatros ciudades argentinas: dos "modernas", Buenos Aires y Córdoba; una "tradicional", Corrientes; y otra "transicional", Tucumán.

Dos hechos resaltan en el caso de Santiago del Estero: la exigüidad extrema del empleo industrial —rama 3-y la magnitud de la rama 9 de Servicios: la nota inversa es la característica de Buenos Aires. Por razones en las que confluyen la casi inexistencia de inversiones privadas demandantes de empleo en escalas equiparables al crecimiento natural de la población y el comportamiento clientelístico de sucesivos gobiernos, el sector estatal ha crecido hasta el punto de ocupar cerca de la mitad de la PEA urbana. Es lo que explica la magnitud de la rama 9.

Y por tratarse de empleo estatal — de "puestos públicos"- resulta elevada la participación de la categoría ocupacional de asalariados en dicho sector (Cf. Cuadro 3), al igual que en la otra ciudad "tradicional", Corrientes; en cambio, en las ciudades modernas se verifica una mayor incidencia del cuentapropismo en la rama "Servicios".

Esto significa que, teniendo en cuenta los niveles existentes de productividad e ingresos, en áreas urbanas tradicionales de la Argentina, el empleo público estaría actuando como refugio para los pobres no marginales, es decir para aquellos segmentos de la población que poseen un mínimo básico de instrucción formal. Por otra parte, también tenemos el caso de ciertos profesionales universitarios: para quienes no disponen de una especialidad compatible con el ejercicio liberal o independiente -que no es el caso, por ejemplo, de médicos y abogados - existe una fuerte probabilidad que en las zonas tradicionales deban emplearse en el sector público. En cambio, en las áreas modernas, que por su mayor complejidad social concentran una demanda más alta y más variada de servicios, también presentan una mayor cantidad de opciones ocupacionales en el sector privado.

\section{Cuadro 2}

ESTRUCTURA SECTORIAL DEL EMPLEO EN CINCO CIUDADES

En porcentajes sobre la población ocupada total

\begin{tabular}{|c|c|c|c|c|c|c|c|c|c|}
\hline & & \multicolumn{8}{|c|}{ Ramas de Actividad ${ }^{\mathrm{a}}$} \\
\hline & & 1 & 3 & 4 & 5 & 6 & 7 & 8 & 9 \\
\hline S. del Estero & 100,0 & 1,1 & 8,9 & 1,1 & 8,8 & 18,7 & 4,4 & 3,8 & 47,9 \\
\hline Buenos Aires & 100,0 & 0,6 & 25,9 & 0,6 & 6,6 & 19,7 & 5.7 & 5,9 & 31,9 \\
\hline Córdoba & 100,0 & 0,8 & 19,1 & 0,3 & 9.7 & 21,6 & 5,4 & 4,6 & 34,8 \\
\hline Corrientes & 100,0 & 0,9 & 10.1 & 0,7 & 10,4 & 16,2 & 4,8 & 3,5 & 46,3 \\
\hline Tucumán & 100,0 & 1,7 & 13,5 & 0.3 & 7,3 & 23,2 & 5,1 & 3,4 & 36,1 \\
\hline
\end{tabular}

Fuente: INDEC/EPH (1988)

${ }^{2}$ No se incluye Rama 2 por la exigüidad del empleo en ella. 
Cuadro 3

PROPORCIÓN DE TRABAJADORES CUENTA PROPIA EN LA PEA OCUPADA TOTAL Y EN LOS OCUPADOS DE CADA RAMA

En porcentajes

\begin{tabular}{lccccc}
\hline & \multicolumn{4}{c}{ Trabajadores Cuenta Propia } \\
\cline { 2 - 6 } & & \multicolumn{4}{c}{ Ramas de Actividad ${ }^{\text {al }}$} \\
\cline { 2 - 6 } & PEA $^{\text {b } /}$ & Industria & Construcción & Comercio & Servicios \\
\hline S. del Estero & 22,1 & 22.9 & 42,8 & 39,4 & 15,6 \\
Buenos Aires & 19.6 & 9.5 & 40,1 & 32,1 & 23,1 \\
Córdoba & 28.3 & $1+.0$ & 51.5 & 35,4 & 20,6 \\
Corrientes & 18.8 & 19.5 & 35.5 & 13,5 & 13,6 \\
Tucumán & 19.6 & 13.6 & 32.6 & 30.9 & 15,6 \\
\hline
\end{tabular}

Fuente: INDEC/EPH (1988)

a/Proporción de trabajadores cuenta propia en el total de ocupados de cada rama de actividad.

$\mathrm{b} /$ Proporción de trabajadores cuenta propia en la población ocupada total

En suma, en Santiago del Estero la rama de actividad Servicios se encuentra sobrerepresentada en la PEA; consistentemente, la incidencia de esa rama es menor en las ciudades "modernas" de la comparación.

Coincidiendo con las habituales tipologías de modernidad, el sector manufacturero adquiere singular importancia en Buenos Aires y Córdoba; además, por tener significación los establecimientos medianos y grandes, predomina el empleo formal, es decir, asalariado. Consecuentemente, en Santiago del Estero el empleo global en dicho sector tiene escasa participación y la captación de puestos asalariados no es tan alta: se trata de una débil estructura industrial, con predominio de pequeños establecimientos.

También puede constatarse en el mencionado Cuadro 3 que, en las cinco ciudades de la comparación, la representación de los trabajadores por cuenta propia es relevante en el sector Construcción. Este hecho quizás podría ser parcialmente explicado por la circunstancia de ser la construcción una actividad estructuralmente inestable y fuertemente vinculada a los ciclos económicos y, también, porque en su desempeño la antigüedad de la trayectoria laboral y la capacitación empírica configura una estratificación interna de la mano de obra que es rígidamente estructurada. De ahí que en los recurrentes períodos de desempleo que afectan al sector, es factible que que un aprendiz de albañil —que se encuentra en los estadios iniciales de la trayectoria de capacitación- al ser despedido de un puesto asalariado se autogenere un empleo informal en cualquier otra actividad (el comercio, predominantemente). Pero parece resultar menos probable que acontezca otro tanto con quienes poseen una mayor experiencia de práctica y capacitación -oficiales y semioficiales albañiles-; se puede plausiblemente conjeturar que estos últimos encontrarán más viable, y más conveniente, en caso de despido de un empleo asalariado, desplazarse hacia una función de cuenta propia en la misma actividad de construcción. Asimismo se podría razonar que la existencia de empleo asalariado se encuentra vinculada a un tipo de demanda cuya finalidad es la construcción en términos específicos, en tanto que el empleo independiente o cuenta propia se justificaría también por la construcción específica (en este caso en pequeña escala) pero, sobre todo, por la reparación y 
ampliación. Este segundo tipo de requerimientos expresaría la demanda por parte de sectores de ingresos medios y bajos.

Otro sector que interesa particularmente por su significación en la conformación del empleo urbano es el comercio; en todas las ciudades que estamos examinando continúa en importancia al sector Servicios. Ambos sectores, en Santiago, concentran un poco más del 60 por ciento del total del empleo; además, de cada cien trabajadores cuenta propia de la ciudad cuarenta se desempeñan en el comercio. La relevancia del cuentapropismo en esta actividad no sólo es patrimonio de la ciudad, también lo es en el conjunto de la provincia; una estimación señala que de los 17 mil ocupados en el comercio alrededor de 8 mil eran vendedores ambulantes.

\section{SOBRE LA SUBUTILIZACIÓN LABORAL}

Diversas perspectivas coinciden en señalar que la sola tasa de desempleo abierto subestima el grado de desaprovechamiento de la fuerza de trabajo. Para apreciar más fidedignamente la subutilización laboral es necesario apelar a medidas que digan relación de las diversas modalidades del subempleo (voluntario e involuntario) y de las tasas de actividad vigentes. En el caso de Santiago, se observa en el Cuadro 4, que mientras la tasa de desempleo abierto relativamente se estabiliza - y aún desciende en el último quinquenio-, la tasa de subocupación asciende sostenidamente hasta cerca del 20 por ciento en 1986 y luego se sitúa en alrededor del 13 por ciento. Y si se considera que la tasa de actividad disminuye o se mantiene por debajo del promedio nacional (nótese que son las más bajas, junto a Corrientes) tal vez se podría comenzar a validar la hipótesis que predica que la tasa de desempleo abierto puede ser controlada por el incremento de la tasa de subempleo y por el aumento de la magnitud del desempleo oculto (fuerza laboral censalmente considerada "inactiva", y que en realidad se retira o no desea ingresar al mercado laboral por las bajas remuneraciones ofrecidas) ${ }^{2}$. Es en el marco de la conceptualización sobre el desempleo oculto que adquieren sentido los datos de 1982: es el año en que resulta mayor la tasa de actividad y, consecuentemente, también mayor el valor del desempleo abierto.

La distorsión que supone el uso - como única medida - de la tasa de desempleo abierto, es más seria cuando se la presenta —como es habitual hacerlo- como una tasa general de desempleo, como un valor que dice referencia de una característica global de la PEA. Por ello nos pareció justificado elaborar el Cuadro 5 donde se torna visible la distribución sectorial del desempleo, siendo posible entonces discriminar rasgos más afinados de la desocupación. En las cinco ciudades consideradas, la mayor cantidad de empleo en la rama Servicios explica que sea en ésta donde se concentren valores más altos de desempleo; pero en términos estrictos, la crisis ocupacional se manifiesta más acentuadamente en el sector manufacturero en las jurisdicciones desarrolladas (que es el caso de Buenos Aires), en tanto que en las áreas rezagadas se manifiesta en las actividades tradicionales (que es el caso del comercio en Santiago). Así, en Buenos Aires de cada 100

\footnotetext{
${ }^{2}$ Una conceptualización sobre el desempleo oculto fue desarrollada por Alvaro Orsatti en sus trabajos en el CELL/CONICET.
} 
TRABAJO PRECARIO Y DESEMPLEO OCULTO: LA ESTRUCTURA OCUPACIONAL EN...

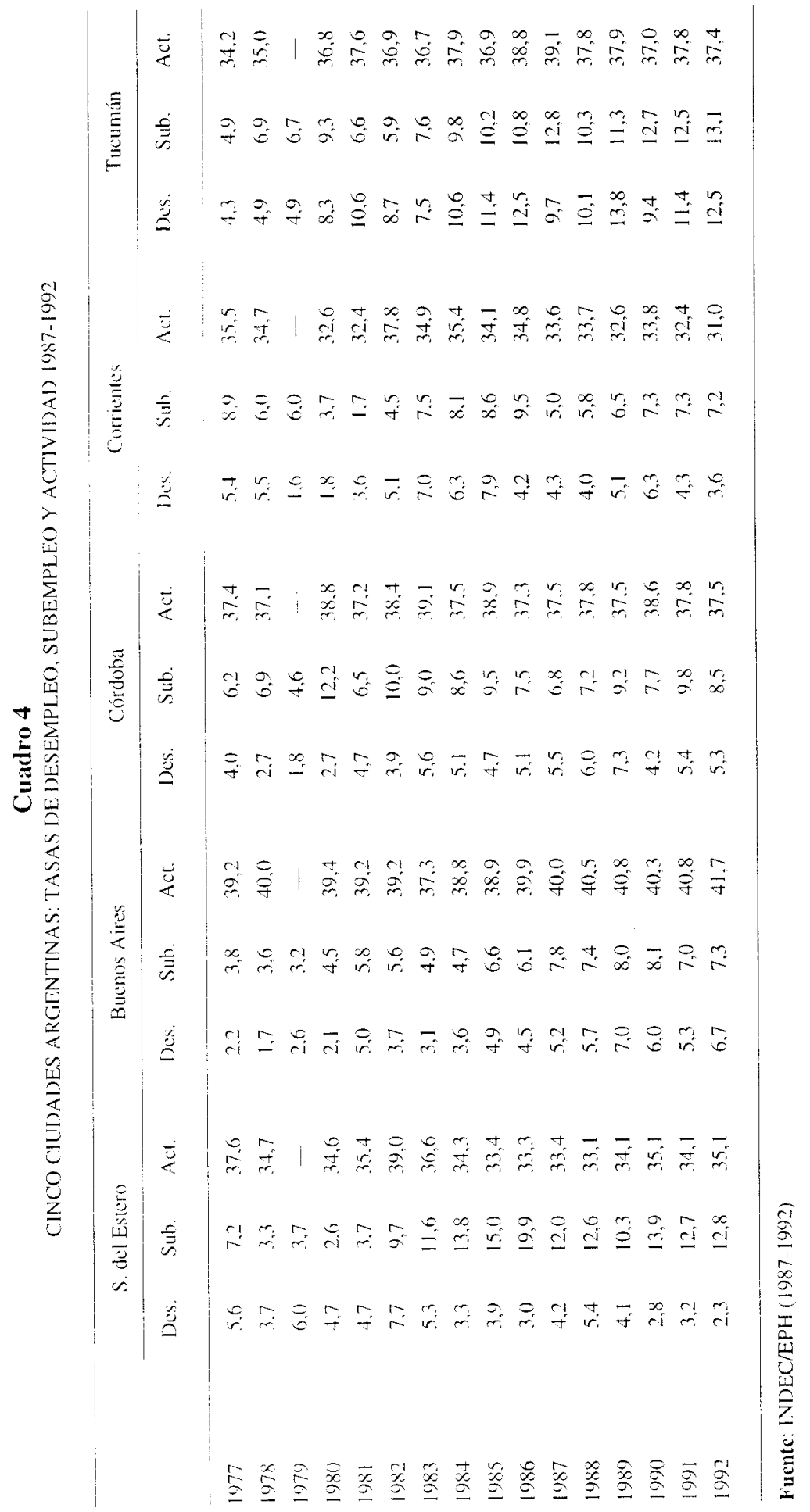


REVISTA DE SOCIOLOGÍA

Cuadro 5

TASAS SECTORIALES DE DESEMPLEO EN CINCO CIUDADES

\begin{tabular}{lcccc}
\hline & \multicolumn{4}{c}{ Desempleo por rama de actividad } \\
\cline { 2 - 5 } & Industria & Construcción & Comercio & Servicios \\
\hline S. del Estero & 6,2 & 8,3 & 18,8 & 20,8 \\
Buenos Aires & 16,4 & 11,5 & 11,9 & 22,9 \\
Córdoba & 17,2 & 13,8 & 17,9 & 18,5 \\
Corrientes & 12,8 & 16,6 & 13,7 & 29,5 \\
Tucumán & 13,3 & 14,6 & 17,2 & 17,5 \\
\hline
\end{tabular}

Fuente: INDEC/EPH (1988)

${ }^{\text {a }}$ La tasa total de desempleo de cada jurisdicción es $=100.00$

desocupados, 23 lo son de servicios y 16 de la industria; pero en Santiago 21 lo son de servicios y 19 del comercio, en tanto que sólo 6 de la industria.

\section{EL NIVEL EDUCATIVO DE LA POBLACIÓN OCUPADA}

Puede resultar revelador inspeccionar las características del nivel educativo de la PEA en Santiago del Estero. Se comprueba que los grados de instrucción de los ocupados son superiores a los de la población desempleada: se torna visible, sobre todo, en los ciclos de instrucción "completa". Un proceso similar se constata en Buenos Aires - como se ve en el Cuadro 6-, y, se puede decir, en todos los mercados urbanos de la Argentina.

Evidencias semejantes han sido registradas en otros contextos latinoamericanos (CEPAL/Unesco, 1984 y Bélisie, 1992). Pero una característica específica de la Argentina es la proporción de universitarios que se encuentran desempleados; la crisis iniciada en los años 80 ha agravado en el país la estructural incongruencia entre la sobreoferta del subsistema educativo y la demanda efectiva del sistema productivo.

La distribución de la PEA urbana de Santigo del Estero, por rama de actividad y clasificada según nivel de instrucción, como se registra en el Cuadro 7, permite sustentar determinadas conjeturas sobre los grados de modernidad de las distintas áreas productivas a partir de los niveles de calificación que demanda cada una de ellas.

Según esta perspectiva, una actividad resulta nítidamente tradicional: la construcción. Ella concentra la mayor proporción de trabajadores con tan sólo instrucción primaria $y$, correlativamente, los requerimientos más exiguos de personal universitario. Como ya se señaló, el empleo en la construcción, en Santiago del Estero, está generado por muy pocos establecimientos de grandes dimensiones, por un número de microempresas y, sobre todo, por trabajadores cuenta propia. Algunos de estos rasgos se repiten en el sector manufacturero, pero es mayor la captación de empleo de trabajadores con instrucción secundaria.

Contrapuestas a la anterior son las características de las ramas 8 -Finanzas y seguros- y 4 -Electricidad, gas y agua-; porque comprenden actividades que se realizan casi excluyentemente en establecimientos del sector formal que poseen una demanda de calificación más alta, algo que se torna ostensible en el sector finanzas que recluta casi la mitad de su fuerza de trabajo entre la población con estudios universitarios. 
TRABAJO PRECARIO Y DESEMPLEO OCULTO: LA ESTRUCTURA OCUPACIONAL EN...

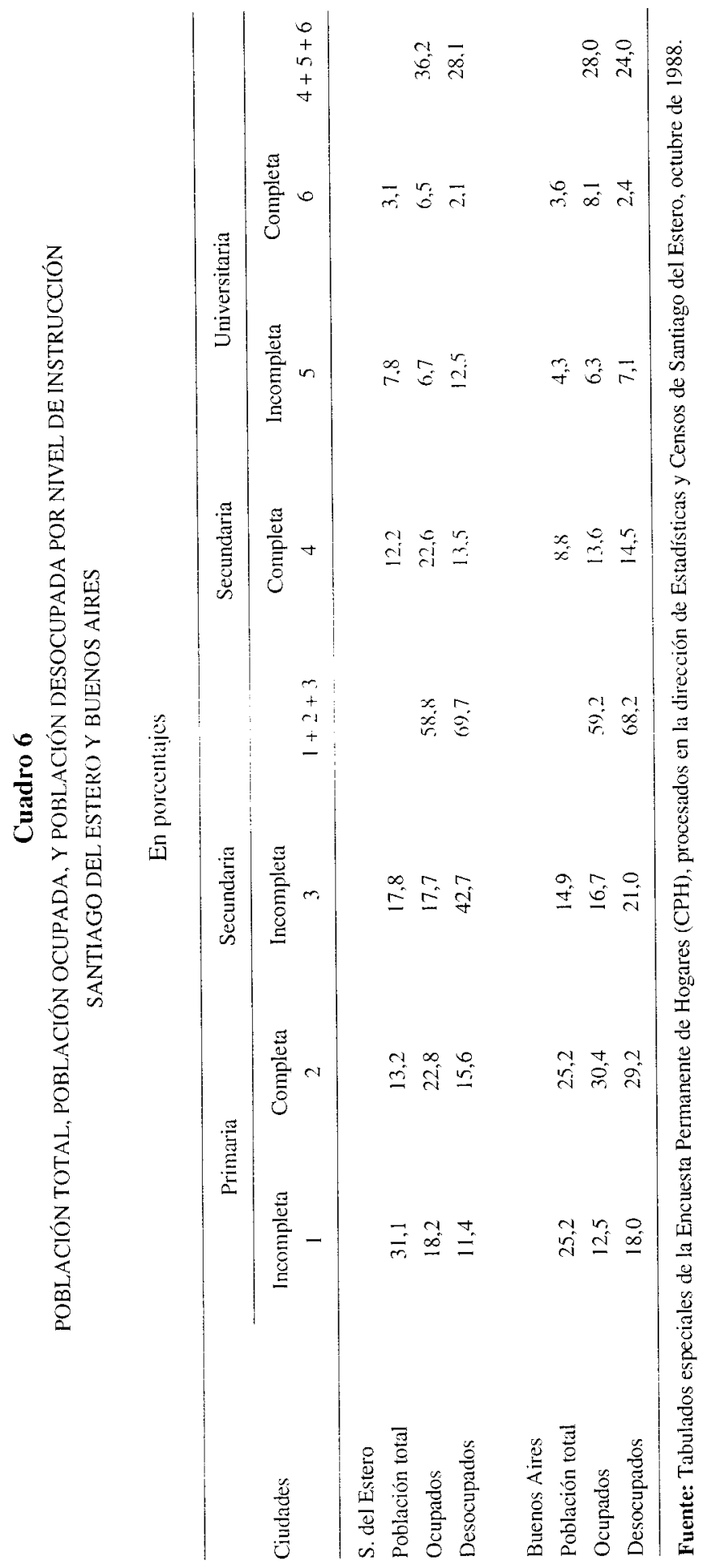


REVISTA DE SOCIOLOGÍA

Cuadro 7

POBLACIÓN ECONÓMICAMENTE ACTIVA POR RAMA DE ACTIVIDAD

Y NIVEL DE INSTRUCCIÓN. SANTIAGO DEL ESTERO

En porcentajes

\begin{tabular}{lcccccccc}
\hline & \multicolumn{9}{c}{ Ramas de Actividad $^{\mathrm{a}}$} \\
\cline { 2 - 8 } $\begin{array}{l}\text { Nivel de } \\
\text { instrucción } \mathrm{b}\end{array}$ & 3 & 4 & 5 & 6 & 7 & 8 & 9 \\
\hline Primario & 51,4 & 30,9 & 73,7 & 39.7 & 48.9 & 5,9 & 47,6 \\
Secundario & 41,9 & 38,4 & 17,5 & 47,9 & 37,2 & 47.3 & 33,9 \\
Terciario & 6,6 & 30,5 & 3,7 & 10,4 & 13,9 & 46.8 & 15,2 \\
& 100,0 & 100,0 & 100,0 & 100,0 & 100,0 & 100,0 & 100,0 \\
\hline
\end{tabular}

Fuente: Idem Cuadro 6.

aNo se consignan Ramas 1 y 2 por consignar muy pocos datos.

${ }^{b}$ En este caso el procesamiento de origen no discrimina niveles educativos completo e incompleto.

Y en una situación transicional, entre la rezagada construcción y las más desarrolladas banca y energía, se encuentran las ramas de Comercio y Servicios. Estas dos últimas, conjuntamente, captan las tres cuartas partes del total del empleo urbano, y aunque poseen algunas características comunes - $\mathrm{vg}$. similares requerimientos de empleo con instrucción primaria-, sin embargo genéricamente pertenecen, por así decir, a universos distintos: uno, el sector servicios, al mundo formal ${ }^{3}$, y la otra, la rama comercio, predominantemente al sector informal. Entre otras razones porque, como ya se advirtió, en Santiago del Estero el cuentapropismo implica el 40 por ciento del total del empleo comercial, en tanto que los asalariados representan el 80 por ciento del sector servicios. En rigor, de cada cien universitarios que trabajan cincuenta y ocho lo hacen en servicios, proporción mayor al peso del empleo en servicios en la PEA total que es del 50 por ciento.

\section{LOS INGRESOS: EDUCACIÓN Y SEXO}

La heterogeneidad y segmentación de la fuerza laboral santiagueña se visualiza con nitidez en los distintos niveles de ingreso que son generados por la distribución social de la educación.

No obstante que la información que se transcribe en el Cuadro 8 no ha podido ser desagregada aún más — porque los procesamientos de la EPH no discriminan, para este caso de cruce con la variable ingresos, entre nivel educativo completo e incompleto- de todos modos ella resulta elocuente: existe una muy fuerte correlación entre la distribución del ingreso y el nivel educativo. Quienes no han superado el nivel educativo primario se agrupan mayoritariamente en los tres tramos inferiores de ingresos -el 56.7 por ciento- y sólo representan el 18 por ciento en las escalas más altas de ingreso. A la inversa,

\footnotetext{
La formalidad del sector servicios se mantiene aún teniendo en cuenta que en él está incluido el servicio doméstico asalariado. En 1992. por ejemplo. de 30.994 asalariados en la rama 9, 2.348 son domésticas asalariadas. sólo el 7.5 por ciento.
} 


\section{Cuadro 8}

POBLACIÓN ECONÓMICAMENTE ACTIVA POR ESCALA DE INGRESOS Y NIVEL DE INSTRUCCIÓN EN SANTIAGO DEL ESTERO

En porcentajes

\begin{tabular}{|c|c|c|c|c|c|c|}
\hline \multirow[b]{2}{*}{ Tramos de Ingreso } & \multicolumn{6}{|c|}{ Nivel de Instrucción ${ }^{a}$} \\
\hline & Primario & & Secundario & & Terciario & \\
\hline 1 & 20,3 & & 6.4 & & 1,2 & \\
\hline 2 & 16,3 & & 7.2 & & 4,7 & \\
\hline \multirow[t]{2}{*}{3} & 20.1 & & 18.4 & & 13,3 & \\
\hline & $\rightarrow$ & 56,7 & $\rightarrow$ & 32,0 & $\rightarrow$ & 8,4 \\
\hline 4 & 8,4 & & 13.5 & & 5,2 & \\
\hline \multirow[t]{2}{*}{5} & 6,7 & & 10.3 & & 5,8 & \\
\hline & $\rightarrow$ & 5.1 & $\rightarrow$ & 23,6 & $\rightarrow$ & 11.0 \\
\hline 6 & 12,1 & & 17.3 & & 16,4 & \\
\hline 7 & 4.2 & & 7.4 & & 15,7 & \\
\hline \multirow[t]{2}{*}{8} & 2.1 & & 9.1 & & 29,7 & \\
\hline & $\rightarrow$ & 8.4 & $\rightarrow$ & 33,8 & $\rightarrow$ & 61,8 \\
\hline
\end{tabular}

Fuente: Idem Cuadro 6.

aEl procesamiento no discrimina niveles de instrucción Completo e Incompleto.

más del 60 por ciento de los ocupados universitarios se sitúan en los tres tramos superiores de ingreso.

Los perceptores con estudios secundarios ocupan, en cambio, una situación intermedia y poseen una mayor dispersión que los grupos anteriores.

Por otra parte, diversas conceptualizaciones suelen predicar la existencia de mayores niveles salariales entre los ocupados formales que en los trabajadores por cuenta propia. Tal postulado también se cumple en la Argentina. Y, además, se le puede añadir una certidumbre: cuanto más atrasada - más heterogénea - es una estructura productiva, mayores son los diferenciales de ingreso entre las categorías ocupacionales.

En todas las ciudades consideradas - como se advierte en el Cuadro 9-, los tramos superiores de ingreso congregan una proporción mayor de asalariados que de mano de obra independiente.

Finalmente, se comprueba que en Santiago del Estero, como acontece en otros contextos, el desempleo es mayor entre las mujeres que entre los varones (Cuadro 10). Pero, además, los ingresos de la población femenina ocupada son sustancialmente inferiores a los correspondientes a los ocupados masculinos. Los cuatro tramos más bajos de ingreso reúnen al 70 por ciento de las mujeres y sólo al 46 por ciento de varones (ver Cuadro 11). La mayor frecuencia femenina se verifica en el tramo más bajo: acontece que de las ocho mil mujeres en ese tramo el 51.8 por ciento son empleadas domésticas asalariadas ("puertas adentro", es decir relevadas como componentes del hogar empleador).

\section{ACOTACIONES SOBRE LAS ENTREVISTAS}

En este apartado se presentan algunas consideraciones referidas a entrevistas efectuadas 
REVISTA DE SOCIOLOGÍA

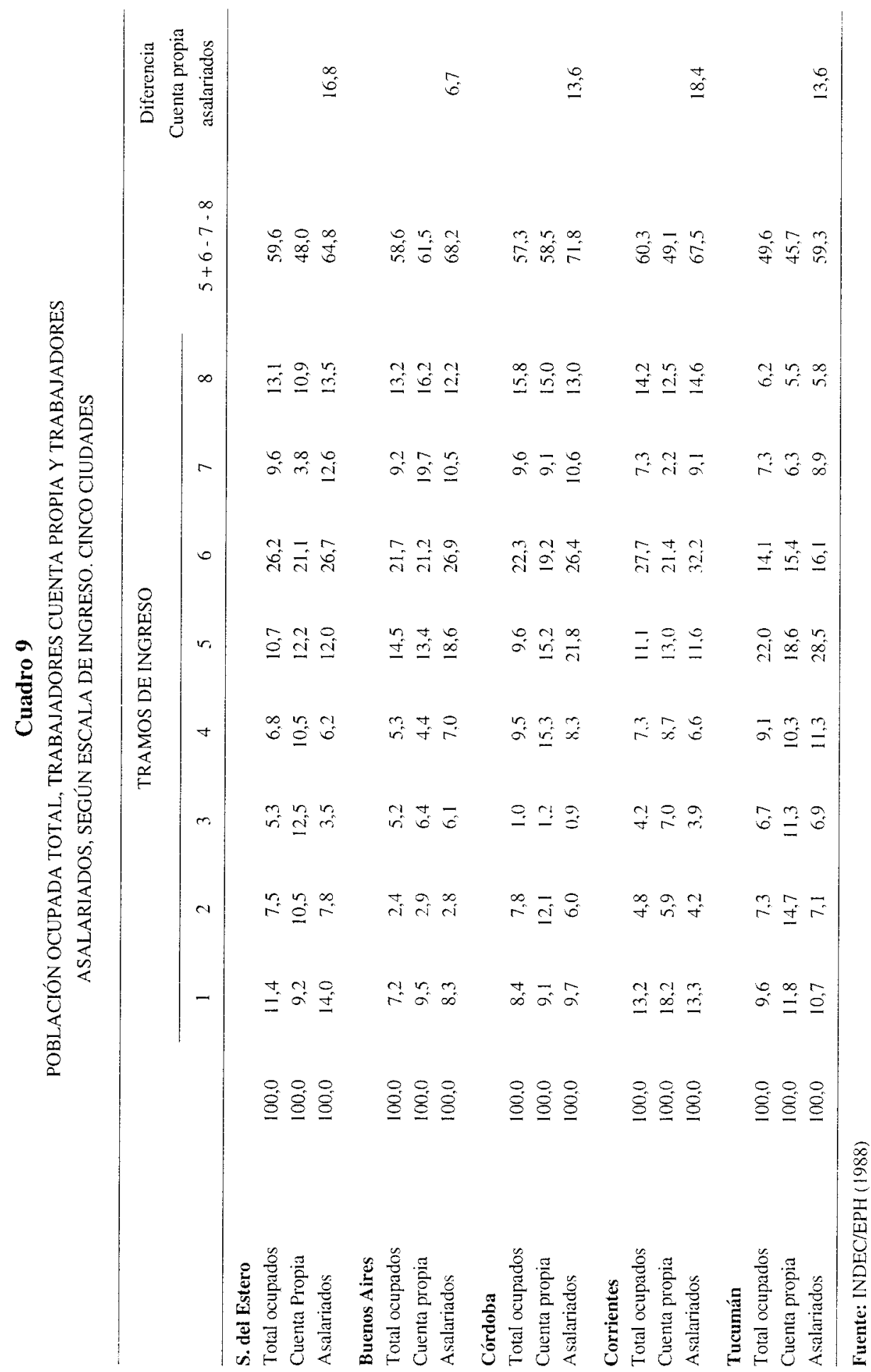


TRABAJO PRECARIO Y DESEMPLEO OCULTO: LA ESTRUCTURA OCUPACIONAL EN...

\section{Cuadro 10}

TASAS DE DESEMPLEO Y ACTIVIDAD POR SEXO

EN CINCO CIUDADES

\begin{tabular}{lcccccc}
\hline & \multicolumn{2}{c}{ Desempleo } & & \multicolumn{2}{c}{ Actividad } \\
\cline { 2 - 3 } \cline { 5 - 7 } Ciudades & Varones & \multicolumn{2}{c}{ Mujeres } & & Varones & Mujeres \\
\hline S. del Estero & 6,0 & & 11.2 & & 47,1 & 29,8 \\
Buenos Aires & 5,0 & 5.7 & & & 53,5 & 24,2 \\
Córdoba & 4,0 & 5.2 & & & 52,5 & 24,8 \\
Corrientes & 7,1 & 7.3 & & & 48,4 & 25,4 \\
Tucumán & 6,2 & & 11.7 & & 49.2 & 25,9 \\
\hline
\end{tabular}

Fuente: INDEC/EPH (1988)

\section{Cuadro 11}

POBLACIÓN OCUPADA POR SEXO

SEGÚN ESCALA DE INGRESOS EN CINCO CIUDADES

En porcentajes

\begin{tabular}{|c|c|c|c|c|c|c|}
\hline Escala de Ingresos & Varones & & Mujeres & & Total & \\
\hline 1 & 3.3 & & 25,9 & & 13,2 & \\
\hline 2 & 10,1 & & 14,7 & & 12,1 & \\
\hline 3 & 20.8 & & 19,6 & & 19,9 & \\
\hline \multirow[t]{2}{*}{4} & 12,4 & & 9,4 & & 11,1 & \\
\hline & $\rightarrow$ & 46,6 & $\rightarrow$ & 69.5 & $\rightarrow$ & 56,3 \\
\hline 5 & 10,9 & & 6,5 & & 9,1 & \\
\hline 6 & 20,1 & & 11,1 & & 15,9 & \\
\hline 7 & 8.2 & & 7,3 & & 8,0 & \\
\hline \multirow[t]{2}{*}{8} & 13,2 & & 5.2 & & 9,9 & \\
\hline & $\rightarrow$ & 52,1 & $\rightarrow$ & 30,3 & $\rightarrow$ & 42,9 \\
\hline
\end{tabular}

a trabajadores en actividades informales y, a título ilustrativo, se ofrecen breves comentarios sobre dos de las actividades, el comercio y el servicio doméstico. Ellas fueron realizadas a treinta y cinco trabajadores informales, seleccionados de un listado mayor en virtud de criterios de accesibilidad técnica y humana, y, sobre todo, por parecer que representaban ejemplos prototípicos de casos de trabajo informal que interesaban estudiar. A tal efecto, se tuvo en cuenta las acotaciones de Bertaux sobre el tamaño óptimo de una muestra representativa en estudios cualitativos (estrategia del conocimiento por saturación).

A los casos de actividad informal que representan los trabajadores entrevistados es posible clasificarlos según el esquema sugerido por Montaño (1985) en un análisis sobre México. Se proponen así cuatro niveles de análisis:

i) unidades informales de refugio o residuales, que producen muy exiguos márgenes de utilidad por poseer una productividad muy reducida; comprenden servicios de distribución ambulante, algunos servicios personales (limpiaparabrisas, lustrabotas, etc.), el servicio doméstico, y otras actividades (ropavejero, lanzallamas, etc.) 
ii) unidades informales subordinadas, que son actividades que están limitadas en su expansión no tanto por razones de baja productividad, sino por estar subordinadas a establecimientos del sector formal (la subcontratación o la maquila en México)

iii) unidades informales complementarias, que "operan sobre mercados complementarios a los dominados por las empresas del sector formal, actuando en mercados específicos, enfocados hacia las necesidades de ciertos sectores sociales y aprovechando su capacidad de especialización y flexibilidad": las microempresas industriales, el pequeño comercio establecido, la preparación y venta de alimentos en pequeña escala, y los servicios de reparación (sobre todo de automóviles y motocicletas)

iv) unidades informales competitivas, que agrupan actividades dirigidas a mercados restringidos que no resultan rentables para la gran empresa; se trata de actividades artesanales que requieren alta habilidad manual, pero que poseen formas de organización inadecuadas, y que por sus altos costos de producción impiden ingresos mayores. Comprende actividades artesanales en general y "confecciones de sastrería y costura a particulares".

El esquema propuesto por Montaño fue adoptado para el estudio sobre Santiago del Estero; pero no se utilizó el nivel de las unidades subordinadas por comprender actividades de casi ninguna significación en la ciudad. La "maquila" -rasgo típico de la heterogeneidad productiva - se encuentra asociada al funcionamiento de una gran actividad industrial en el sector formal, que no es el caso de Santiago. En realidad, la maquila, de gran importancia en países del tercer mundo (México, entre otros) no posee similar relevancia en Argentina, aunque hayan sido revelados casos de subcontratación en la industria textil en Buenos Aires (Schmukler, 1976) y en las autopartes en Córdoba 4 .

Con estas salvedades, se pueden clasificar las actividades registradas de la siguiente manera:

a) Unidades de refugio: 4 vendedores ambulantes y 2 empleadas domésticas

b) Unidades complementarias: 2 industriales, 3 comerciantes, 2 mecánicos, 4 reparadores, 2 albañiles, 1 taxista, 1 peluquera, 1 apicultor

c) Unidades competitivas: 1 modista, 12 artesanos $^{5}$.

Sobre los trabajadores en unidades de refugio son pertinentes las siguientes consideraciones:

\section{- Vendedores ambulantes}

Por efectos de la crisis económica, en los últimos años ha sido frecuente observar, en casi todas las ciudades argentinas, la aparición de contingentes de vendedores ambulantes. Si bien la venta callejera aún no posee en el país la magnitud e importancia que tiene en otros países de la región - aún no existe, en cuanto a masividad, algo similar a los vastos "tianguis" mexicanos, los "mercados informales" de Lima o las "ferias persas" de Santiago de Chile - la significación económica de la actividad es de creciente importancia, ya lo era en 1987 según lo demuestra un estudio (INDEC, 1987) y se puede estimar que,

\footnotetext{
${ }^{4}$ Agradezco a Francisco Giner de los Ríos, del CEE de El Colegio de México, sus sugerencias en este aspecto.

${ }^{5}$ La sobrerrepresentación de artesanos en la muestra se explica por ser un segmento que interesa particularmente en el proyecto original de este trabajo, que contempla la formulación de líneas de acción en apoyo de las unidades competitivas. especialmente en materia de organización y capacitación.
} 
en virtud de lo que parece será el comportamiento de la economía en el mediano plazo, dicho sector se incrementará. Según el ya clásico estudio de De Soto (1988) el comercio ambulante admite dos maneras — que, además, son secuenciales-: el comercio "itinerante" y el que se efectúa "con lugar fijo en la vía pública". Los cuatro vendedores entrevistados (de entre 25 y 45 años, dos con estudios primarios completos y dos incompletos) pertenecen al segundo segmento y todos realizan sus actividades en los alrededores del Mercado Central "Armonía" de la ciudad, donde existe una gran concentración de ambulantes, localización que es frecuente en la mayoría de las ciudades latinoamericanas. Se trata de tres varones (un vendedor de "choripán", uno de empanadas y otro de baratijas importadas) y una mujer vendedora de pescado. Todos cuentan con permiso municipal y pagan sus correspondientes derechos. Llama la atención que en los cuatro casos se verifican situaciones de doble empleo, tanto en el sector formal como en el informal: el vendedor de "choripán" y el de baratijas desempeñan al mismo tiempo ocupaciones asalariadas de bajo nivel en el sector público municipal (donde hay una gran cantidad de asalariados precarios o "contratados"); el vendedor de empanadas también es albañil por cuenta propia, y la vendedora de pescados trabaja como doméstica por horas. En este último caso se debe acotar que quien la proveee de mercancía para la venta es su esposo - quien pesca a veinte kilómetros de la ciudad dos veces a la semana- que se desempeña como asalariado municipal en recolección de residuos.

\section{- empleadas domésticas}

En otro trabajo (Zurita, 1983) nos referimos con detenimiento al caso de las domésticas de Santiago del Estero. Por diversas razones - que tienen que ver con la pervivencia de pautas señoriales por el lado de la demanda, con una sobreoferta de fuerza de trabajo femenina joven y con la restricción de acceso al sector formal - la ciudad que estamos estudiando es la que proporcionalmente capta la mayor cantidad de empleo doméstico en el país, y tal magnitud se concentra en los estratos más tradicionales del sector, es decir, en el servicio doméstico sin retiro o "puertas adentro". Pero en las dos entrevistadas (una de 35 y la otra de 29 años, ambas con estudios primarios completos) ninguna de ellas reside en el domicilio de la familia empleadora: una, trabaja por horas en tres residencias distintas, y la otra media jornada en una sola familia. ¿La primera sería cuenta propia y la segunda asalariada? En un documento de 1983 tratamos de responder a los interrogantes que se plantean con referencia a la definición de las categorías ocupacionales entre las domésticas. Con todo, se debe señalar que la doméstica cuenta propia entrevistada capta por hora trabajada un ingreso superior a la asalariada, aunque ésta recibe alimentación en el domicilio empleador, y no así la primera. A las dos se les paga vacaciones y aguinaldo, pero a ninguna se le realizan aportes jubilatorios ni para el seguro social. Las percepciones de ambas son muy exiguas. El esposo de la cuenta propia es trabajador independiente gastronómico y el de la segunda es sereno en un edificio público.

\section{OBSERVACIONES FINALES}

A través del examen de su estructura ocupacional, Santiago del Estero muestra la fisonomía característica de las sociedades estancadas. La oferta de puestos de trabajo industriales es exigua, en tanto que es muy alta la captación de empleos públicos en virtud 


\section{REVISTA DE SOCIOLOGÍA}

de un proceso en el que convergen el clientelismo político y la falta de inversión privada; se podría decir que el empleo estatal opera como un refugio social para los "pobres no marginales".

Más significativo que el aumento de la tasa de desempleo abierto es el incremento del subempleo. Además, los bajos niveles de la tasa de actividad estarían expresando el crecimiento de la magnitud del desempleo oculto.

Existen ramas de actividad marcadamente tradicionales como la construcción y el comercio, donde predominan el cuentapropismo y la realización de actividades informales de subsistencia.

También han resultado visibles los condicionamientos que impone el nivel educativo en los procesos de inserción laboral: mayores niveles de instrucción sustentan una probabilidad mayor de obtener empleo; y si así fuera el caso, una mejor educación está asociada a más altos ingresos. Por otra parte, en Santiago del Estero se acentúa un rasgo básico de la Argentina: existe una sobreoferta de personal universitario, lo que no deja de ser curioso en la provincia que presenta uno de los índices más considerables de analfabetismo rural.

Otra evidencia: los trabajadores del sector formal perciben ingresos superiores a los captados por las categorías ocupacionales vinculadas al sector informal, como la cuenta propia.

La nota final la constituye una manifestación de los roles de género en la estructura cupacional: es mayor el desempleo entre las mujeres y, asimismo, en la población ocupada la mano de obra femenina globalmente percibe ingresos inferiores a los varones ocupados.

\section{REFERENCIAS BIBLIOGRÁFICAS}

BELISIE. F. (Comp.) (1992), Trabajo informal y pobreza urbana en América Latina, International Development Research Centre.

Cepal/anesco (1988), La educación en América Laina, Santiago, Chile.

DE Soto. H. (1988), El otro sendero, Diana.

INDEC/EPH (1988 y 1993), Instituto Nacional de Estadísticas y Censos/Encuesta Permanente de Hogares, Tabulados inéditos.

Montaño, J. (1985), Barretas institucionales de entrada al sector informal en la ciudad de México, PREALC.

SCHMUKLER, B. (1978), Relaciones actuales de produccion en industrias tradicionales argentinas, CLACSO.

Tasso. A y C.V. Zurita (1981), Un análisis de la estructura social de Santiago del Estero, Instituto Central de Investigaciones Científicas de la Universidad Católica de Santiago del Estero (INCIC/LCSE).

ZuRTA, C.V. (1983). El servicio doméstico en Argentina: El caso de Santago del Estero, (INCICluCSE)

(1988). Mercado de trabajo, sector informal y politicas de empleo en ciudades intermedias. El caso de Santiago del Estero, Argentina, Centro de Estudios Sociológicos, El Colegio de México. 